\title{
Adjuvant Antibody Therapy with Trastuzumab in Early Breast Cancer
}

\author{
Michael Untch $^{\mathrm{a}^{*}} \quad$ Anton Scharl $\mathrm{b}^{\mathrm{b}^{*}} \quad$ Christian Jackisch ${ }^{\mathrm{c}^{*}} \quad$ Christoph Thomssen $^{\mathrm{d}^{*}} \quad$ Ulrike Nitz $^{\mathrm{e}^{*}}$ \\ Gunter von Minckwitz ${ }^{f^{*}} \quad$ Nadia Harbeck M $^{*}$ \\ a Brustzentrum, Helios Klinikum Berlin-Buch, \\ b Frauenklinik Amberg, \\ ${ }^{c}$ Frauenklinik Offenbach, \\ d Universitätsfrauenklinik Halle (Saale), \\ e Universitäts-Frauenklinik Düsseldorf, \\ ${ }^{f}$ GBG Forschungs GmbH, Neu-Isenburg, \\ ${ }^{g}$ Frauenklinik der Technischen Universität München, Germany
}

\section{Key Words}

HER2/neu - Monoclonal antibody - Breast cancer .

Trastuzumab · Adjuvant therapy

\section{Summary}

Immunotherapy with the monoclonal antibody trastuzu$\mathrm{mab}$ is a well established cornerstone of an individualized treatment strategy for women with HER2/neu-overexpressing breast cancer. Based on the reported data of clinical studies comprising more than 14,000 women, trastuzumab was approved throughout the European Union in May 2006 for adjuvant therapy in patients with early-stage HER2/neu-overexpressing breast cancer.

\section{Introduction}

Approximately $20-25 \%$ of all primary breast cancers show strong overexpression of the human epidermal growth factor HER2/neu, a transmembraneous tyrosine kinase receptor which is part of the HER family of receptors. HER2/neu overexpression is associated with decreased disease-free and overall survival [1] indicating a key role of HER2/neu in breast cancer pathogenesis and progression. Owing to its high incidence and its accepted prognostic and predictive value, HER2/neu became part of the St. Gallen consensus risk classification system in 2005 where patients with HER-overexpressing tumors were classified as being at increased risk [2].

*As members of the Breast Commission, part of the AGO (Working Group Gynecologic Oncology, German Society of Obstetrics and Gynecology)

\author{
Schlüsselwörter \\ HER2/neu · Monoklonaler Antikörper · Mammakarzinom · \\ Trastuzumab · Adjuvante Therapie
}

\section{Zusammenfassung}

Die gegen die HER2/neu-Überexpression gerichtete Immuntherapie mit dem monoklonalen Antikörper Trastuzumab ist ein wesentlicher Bestandteil des individualisierten Behandlungskonzepts bei Patientinnen mit HER2/neu-positivem Mammakarzinom. Vor dem Hintergrund der im Folgenden dargestellten Daten klinischer Studien mit mehr als 14000 Frauen wurde Trastuzumab im Mai 2006 europaweit auch für die adjuvante Behandlung von HER2/neu-positivem Brustkrebs zugelassen.

\section{Immunotherapy with Monoclonal Antibody Trastuzumab}

Breast cancer patients with HER2/neu-overexpressing tumors benefit from therapy using the recombinant humanized monoclonal antibody trastuzumab (Herceptin ${ }^{\circledR}$, Roche Pharma AG, Grenzach-Whylen, Germany) targeting the HER2/neu receptor. Trastuzumab is directed against the extracellular domain of HER2/neu and consequently blocks signal transduction of proliferation signals and cell growth of HER2/neu-overexpressing breast cancer cells ('targeted therapy'). Since August 2000, this monoclonal antibody is approved for therapy of metastatic HER2/neu-positive breast cancer as a monotherapy or in combination with paclitaxel or docetaxel. In May 2006, trastuzumab was approved throughout the European Community for adjuvant therapy of HER2/neu-positive breast cancer following surgery, standard (adjuvant or neoadjuvant) chemotherapy and radiotherapy (if indicated).

Prof. Dr. med. Michael Untch

Frauenklinik/Interdisziplinäres Brustzentrum

Helios Klinikum Berlin-Buch, Akademisches LK der Universität Charité

Wiltbergstr. 50, 13125 Berlin, Germany

Tel. +49 30 9401-2275/-2270, Fax -4326

E-mail muntch@berlin.helios-kliniken.de 


\section{Determination of HER2/neu Status}

Because of the high incidence of HER2/neu overexpression and the accepted prognostic value of this parameter, HER2/neu status should be determined as early as possible. Moreover, trastuzumab therapy is only effective and thus only approved for tumors with proven HER2/neu overexpression. The most recent 2006 guidelines of the AGO (Arbeitsgemeinschaft Gynäkologische Onkologie e.V.) recommend HER2/neu testing in all primary breast cancers using standardized and quality controlled immunohistological and/or molecular methods at presentation [3].

Positive HER2/neu status is either determined as proteine overexpression using immunohistochemistry (IHC) and/or as gene amplification using fluorescence in-situ hybridization (FISH). Today, the recommended method is immunohistochemical staining using the HercepTest ${ }^{\mathrm{TM}}$ (DAKO, Glostrup, Denmark; Oxford 1b, AGO recommendation ++) [3]. A HercepTest HER2/neu score of $3+$ is considered as positive, a score of 0 or $1+$ as negative. Weaker overexpression measuring 2+ should be confirmed by a further FISH test which detects the number of HER2/neu gene copies. Gene amplification with more than 2 gene copies/cells is considered HER2/neupositive. Other determination methods such as polymerase chain reaction (PCR), chromogene in-situ hybridization (CISH) and enzyme-linked immunosorbent assay (ELISA) are not considered to be routine alternatives to IHC and FISH testing to be used as a basis of trastuzumab therapy decisions, and should for now only be used in studies.

\section{Clinical Trials Regarding Adjuvant Trastuzumab Therapy}

The European approval of adjuvant trastuzumab immunotherapy was issued on the basis of a substantial data base of more than 14,000 patients with HER2/neu-positive breast cancer (fig. 1). In particular, it was based on the results of the largest international randomized trial, HERA (HERceptin Adjuvant), with 5,090 patients worldwide from 478 centers in 39 countries. Almost 900 German patients recruited by the large German trial groups AGO (Arbeitsgemeinschaft
Gynäkologische Onkologie), GBG (German Breast Group) and WSG (Westdeutsche Studiengruppe) participated in the HERA trial [4]. In this trial, the percentage of node-negative patients (tumor size $>1 \mathrm{~cm}$ ) was 33\%. In 29\%, 1-3 axillary lymph nodes were involved, and in $28 \%, 4$ and more lymph nodes were involved. The HERA trial evaluated the benefit of either 1 or 2 years of trastuzumab after surgery, standard neoadjuvant or adjuvant chemotherapy (at least 4 cycles) and locoregional radiation therapy (if applicable) versus a standard observation arm without antibody therapy. After a median follow-up of 1 year, data from 3,387 patients from the observation arm and the 1-year treatment arm (planned interim analysis) showed significant improvement of the disease-free survival in the trastuzumab arm (127 events) versus the observation arm $(220$ events $)(p<0.0001)$, which translates into a relative reduction in the relapse risk of $46 \%$. These results were also confirmed by the most recent follow-up analysis after 23 months (ITT; 3-year disease-free survival 80.6 vs. $74.3 \%$; p < 0.0001; fig. 2) [5]. After 2 years, $85.8 \%$ of the trastuzumab-treated patients were relapse-free compared to $77.4 \%$ in the observation arm (hazard ratio 0.54) [4]. More-

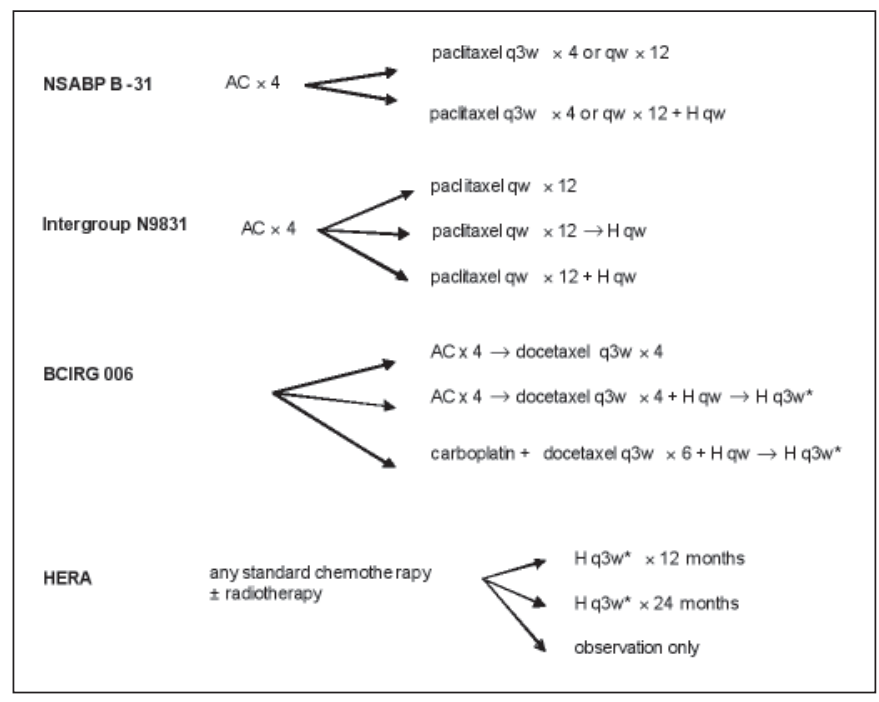

Fig. 1. Overview over large phase III registration trials for adjuvant trastuzumab therapy. $* 6 \mathrm{mg} / \mathrm{kg}$ every 3 weeks.
Fig. 2. Kaplan-Meier curve for a disease-free survival (DFS) and $\mathbf{b}$ overall survival (OS) after adjuvant 1-year trastuzumab-based therapy in the HERA trial (intention-to-treat analysis, median follow-up 2 years) (adapted from [5]).
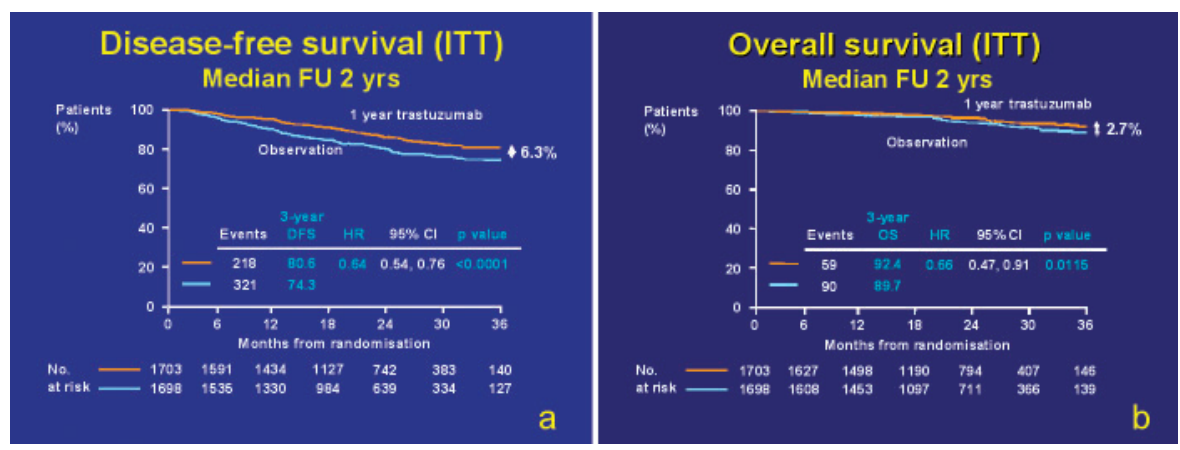
over, relapse-free as well as metastasis-free survival were relative risk reductions of 50 and $49 \%$, respectively (both $\mathrm{p}<0.0001$ ) [4]. The most recent follow-up shows that 1 year of adjuvant trastuzumab therapy also significantly reduces mortality by $34 \%$ (3-year overall survival 92.4 vs. $89.7 \%$; $\mathrm{p}=0.011$ ) and confirms the decreased relapse-risk [5].

The combined interim analysis of the 2 US trials of the National Surgical Adjuvant Breast and Bowel Project (NSABPB 31) and the North Central Cancer Treatment Group (NCCTG-N9831) comprising 3,151 patients confirm the HERA results [6]. In agreement with the FDA (Food and Drug Administration), these 2 trials were analyzed together, since therapy arms and control arms were basically identical (joint interim analysis). Median follow-up at the time of analysis was 2 years (2.4 years in B-31 and 1.5 years in N 9831). The combined analysis was based on 394 events (relapses, metastases, deaths) in 3,151 treated patients. Trastuzumab in addition to the USA standard chemotherapy regimen - doxorubicin/cyclophosphamide (AC) followed by paclitaxel/ trastuzumab $(\mathrm{TH})$ - resulted in an absolute difference in relapse-free survival of $12 \%$ after 3 years and $18 \%$ after 4 years $(\mathrm{p}<0.0001)$ compared to AC followed by paclitaxel alone. In the latter, every third patient experienced a relapse despite standard surgery, radiation therapy and chemotherapy versus only every 6th patient in the trastuzumab arm. The addition of trastuzumab reduced the risk of distant metastases after 3 years by 53\% (absolute difference $8.8 \%$ after 3 years and $15.9 \%$ after 4 years; $\mathrm{p}<0.0001$ ). Adjuvant chemotherapy in combination with trastuzumab resulted in a relative risk reduction regarding overall survival of 33\% (absolute difference $2.5 \%$ after 3 years and $4.8 \%$ after 4 years; $\mathrm{p}=0.015)$.

These results were substantiated by the 3-arm Breast Cancer International Research Group (BCIRG) trial 006 which evaluated the benefit a 1-year trastuzmab therapy either in a sequential therapy of AC, docetaxel +/- trastuzumab (AC_T; $\mathrm{AC} \_\mathrm{TH}$ ) or a simultaneous therapy of a platinum compound, docetaxel and trastuzumab (TCH), in 3,222 women with HER2/neu-positive breast cancer [7]. Median follow-up of 23 months showed a significant superiority of the 2 trastuzumab- containing arms (AC_TH: 77 events, TCH: 98 events) compared to the control arm (AC_T: 147 events) regarding disease-free survival ( $\mathrm{p}<0.0001$ and $\mathrm{p}=0.0002$, respectively). This translated into a risk reduction of $51 \%\left(\mathrm{AC} \_\mathrm{TH}\right)$ and 39\% (TCH), respectively. After 23 months, 93\% (AC_TH) or $91 \%(\mathrm{TCH})$ of the antibody-treated patients were disease-free compared to $86 \%$ of the patients treated with chemotherapy alone (AC_T) (hazard ratio 0.49 and 0.61 , respectively). There was no significant difference in efficacy between the 2 trastuzumab arms $(p=0.16)$. At time of the interim analysis, not enough data for overall survival analysis were available due to the short follow-up period.

\section{Cardiac Safety Profile of Trastuzumab in Adjuvant Therapy}

Also in the adjuvant setting, therapy with the monoclonal antibody was well tolerated. The incidence of cardiac events in the US NCCTG N9831 trial was $2.2 \%$ in the sequential arm (chemotherapy followed by trastuzumab) and $3.3 \%$ in the immuno-chemotherapy combination arm, versus $0 \%$ in the control group [8]. Thus, the rate of cardiac events was lower than the cut-off of $4 \%$ which was pre-defined by an independent data committee before the start of the study. This was also true for the cardiac safety data in the HERA trial where the incidence of cardiac events was only slightly increased in the trastuzumab arm with $0.5 \%$ compared to the $0 \%$ in the control arm. These data were also confirmed by the BCIRG 006 trial which showed an overall low cardiac event rate $(2.62 \%)$ due to trastuzumab therapy (AC_TH). In none of the adjuvant trials, cardiac deaths attributable to trastuzumab therapy were observed. Thus, no increased cardiac danger seems to be present during adjuvant trastuzumab therapy. Nevertheless, close supervision of cardiac function is recommended. AGO recommendations include regular clinical examinations (including electrocardiogram), measurements of weight, heart rate and blood pressure as well as regular evaluation of the left ventricular function by echocardiography before and during trastuzumab therapy [3].
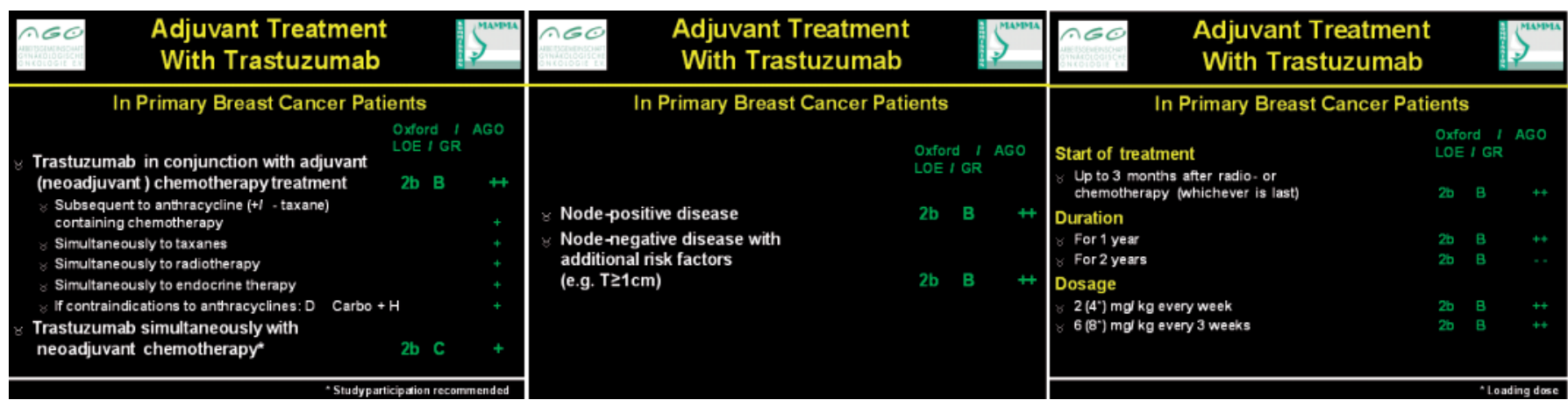

Fig. 3. AGO recommendations for adjuvant trastzumab therapy. 


\section{EU Approval and Most Recent AGO Guidelines Regarding Adjuvant Trastuzumab Therapy}

Trastuzumab is indicated in HER2/neu-positive primary breast cancer for 1 year or until disease progression, whichever comes first. The recommended dosage is a loading dose of $8 \mathrm{mg} / \mathrm{kg}$ body weight followed by $6 \mathrm{mg} / \mathrm{kg}$ every 3 weeks, given intravenously over $90 \mathrm{~min}$. In addition, simultaneous administration following the regimen in the US trials ( $\mathrm{AC}$ followed by taxane + trastuzumab) is specified in the product labeling and thus permitted. This regimen uses weekly administration of the antibody in accordance with the use in the metastatic setting. There are no limitations regarding age, nodal status or time distance to previous therapy. The most recent AGO guidelines recommend adjuvant trastuzumab therapy in patients with HER2/neu-overexpressing breast cancer and nodal involvement as well as in node-negative disease with additional risk factors, such as tumor size $\geq 1 \mathrm{~cm}$ (Oxford 2b; AGO recommendation ++ ) [3]. The antibody therapy can be given with or without a taxane as well as sequentially or simultaneous to the chemotherapy (Oxford $2 \mathrm{~b}$; AGO recommendation ++) [3]. Therapy should be started within 3 months after radiotherapy or chemotherapy and be administered either weekly at a dose of $2 \mathrm{mg} / \mathrm{kg}$ body weight or 3-weekly at $6 \mathrm{mg} / \mathrm{kg}$ (Oxford 2b; AGO recommendation $++)$ [3] (fig. 3).

\section{References}

1 Slamon DJ, Godolphin W, Jones LA, Holt JA Wong SG, Keith DE, Levin WJ, Stuart SG, Udove J, Ullrich A: Studies of the HER-2/neu proto-oncogene in human breast and ovarian cancer. Science 1989;244:707-712.

2 Goldhirsch A, Glick JH, Gelber RD, Coates AS, Thurlimann B, Senn HJ, panel members: Meeting highlights: international expert consensus on the primary therapy of early breast cancer 2005 . Ann Oncol 2005;16:1569-1583.

3 Arbeitsgemeinschaft Gynäkologische Onkologie e.V. (AGO) Organkommission Mammakarzinom (2006): Adjuvante Therapie mit Trastuzumab. www.ago-online.de.

4 Piccart-Gebhardt MJ, Procter M, Leyland-Jones B, Goldhirsch A, Untsch M, Smith I, Gianni L, Baselga J, Bell R, Jackisch C, Cameron D, Dowsett M, Barrios CH, Steger G, Huang CS, Andersson M, Inbar M, Lichinitser M, Lang I, Nitz U, Iwata H, Thomssen C: Trastuzumab after adjuvant chemo- therapy in HER2/neu-positive breast cancer. N Engl J Med 2005;353:1659-16672.

5 Smith I: Trastuzumab following adjuvant chemotherapy in HER2/neu-positive early breast cancer (HERA trial): disease-free and overall survival after 2 year median follow-up. Scientific Special Session, ASCO Annual Meeting 2006, Atlanta/ Georgia, USA

6 Romond EH, Perez EA, Bryant J, Suman VJ, Geyer CE Jr, Davidson NE, Tan-Chiu E, Martino S, Paik S, Kaufman PA, Swain SM, Pisansky TM, Fehrenbacher L, Kutteh LA, Vogel VG, Visscher DW, Yothers G, Jenkins RB, Brown AM, Dakhil SR, Mamounas EP, Lingle WL, Klein PM, Ingle JN, Wolmark N: Trastuzumab plus adjuvant chemotherapy for operable HER2/neu-positive breast cancer. N Engl J Med 2005;353:1673-1684.

7 Slamon D, Eiermann W, Robert N, Pienkowski T, Martin M, Pawlicki M, Chan M, Smylie M, Liu M, Falkson C, Pinter T, Fornander T, Shiftan T, Valero
V, Mackey J, Tabah-Fisch I, Buyse M, Lindsay MA, Riva A, Bee V, Pegram M, Press M, Crown J, on behalf of the BCIRG 006 Investigators: Phase III trial comparing AC-T with AC-TH and with $\mathrm{TCH}$ in the adjuvant treatment of HER2/neu positive early breast cancer patients: first interim efficacy analysis. Breast Cancer Res Treat 2005;94(suppl 1):abstr 1.

8 Tan-Chiu E, Yothers G, Romond E, Geyer CE Jr, Ewer M, Keefe D, Shannon RP, Swain SM, Brown A, Fehrenbacher L, Vogel VG, Seay TE, Rastogi P, Mamounas EP, Wolmark N, Bryant J: Assessment of cardiac dysfunction in a randomized trial comparing doxorubicin and cyclophosphamide followed by paclitaxel, with or without trastuzumab as adjuvant therapy in node-positive, human epidermal growth factor receptor 2-overexpressing breast cancer: NSABP B-31. J Clin Oncol 2005;23:78117819.0 